\title{
Nonlocal response and surface-barrier-induced rectification in Hall-shaped mesoscopic superconductors
}

\author{
D. Y. Vodolazov ${ }^{1,2}$ and F. M. Peeters ${ }^{1, *}$ \\ ${ }^{1}$ Departement Fysica, Universiteit Antwerpen (Campus Drie Eiken), Universiteitsplein 1, B-2610 Antwerpen, Belgium \\ ${ }^{2}$ Institute for Physics of Microstructures, Russian Academy of Sciences, 603950, Nizhny Novgorod, GSP-105, Russia \\ I. V. Grigorieva and A. K. Geim \\ Department of Physics and Astronomy, University of Manchester, Oxford Road, Manchester M13 9PL, United Kingdom
}

(Received 26 October 2004; revised manuscript received 9 March 2005; published 25 July 2005)

\begin{abstract}
Nonlocal response in Hall-shaped superconductors is studied using the time-dependent Ginzburg-Landau equations. Applying current in one pair of contacts leads to a voltage drop in another pair of contacts situated at a distance much larger than the coherence length. This effect is a consequence of the long range correlations in a one-dimensional vortex lattice squeezed in a narrow channel by screening currents. The discrete change in the number of vortices in the channel with applied magnetic field leads to a nonlocal response which is a nonmonotonous function of the magnetic field. For specific configurations of the Hall-shaped superconductor we found a rectifying effect.
\end{abstract}

DOI: 10.1103/PhysRevB.72.024537

PACS number(s): 74.25.Op, 74.20.De, 73.23.-b

In the last decade a number of hybrid superconducting/magnetic ${ }^{1}$ or superconducting ${ }^{2-4}$ structures were experimentally investigated which could be used, e.g., as rectifiers, vortex lenses, and pumps. Nonlocal effects as a consequence of vortex interaction were observed in Corbino disks ${ }^{5,6}$ and in superconducting strips. ${ }^{7}$ There is plenty of theoretical work devoted to the study of the rectify effect in superconducting structures. Various mechanisms leading to rectification were studied. For example in Ref. 8 the superconductor with specially modulated thickness and in Ref. 9 the system with asymmetric channel walls were proposed for controlled motion of the vortices (magnetic flux); numerical calculations were made for the ratchet effect produced by an array of randomly distributed, ${ }^{10}$ and asymmetrically shaped $^{11,12}$ pinning centers. The net motion of one kind of particle under the effect of an ac drive force is also possible if there is a second kind of particle interacting with the first one. ${ }^{13-15}$ Besides, the ratchet effect was experimentally observed in Josephson annular junction with no asymmetry in space but with a time asymmetric ac signal. ${ }^{16}$

In our work we studied the response of a Hall-bar-shaped superconductor to a dc current (see Fig. 1) in the presence of a perpendicular magnetic field. Recently, we found ${ }^{17}$ experimentally a nonlocal response in such a geometry with the superconductor MoGe. Applying a current to one strip (e.g., $A$ to $B$ ) can lead to the appearance of a voltage in the other superconducting strip (e.g., between $C$ and $D$ ). A similar nonlocal effect has been observed previously in mesoscopic semiconducting structures ${ }^{18,19}$ where it was a consequence of the wave character of electrons. Here, the nonlocal effect is a consequence of the current induced deformation of the quasione-dimensional vortex lattice.

Our geometry (see Fig. 1) has the advantage that it allows us to study differences in vortex entry/exit conditions arising from the surface barrier effect. It is impossible to study this by transport measurements in a local geometry because processes of vortex entry and vortex exit are simultaneously present in this case. In our nonlocal geometry we show below that the induced nonlocal voltage arises mainly due to either vortex entry or vortex exit (depending on the sign of the applied current) via the sample edge in the remote lead and it results in the rectification of the applied current. To our knowledge this is the first time that rectification is found through the surface barrier effect.

In our model the external current was applied along the $A B$ lead (Fig. 1) and the nonlocal voltage $V_{\text {nloc }}$ was measured at the $C D$ lead. The nonzero voltage observed along the $C D$ lead is the result of the interaction of the vortices with the applied current in the strip $A B$ and the interaction of the vortices in strip $C D$ with the vortices in the superconductor $A B$ through the vortices localized in the connecting strip. In our calculations we used the time-dependent GinzburgLandau equations

$$
\begin{gathered}
u\left(\frac{\partial \psi}{\partial t}+i \varphi \psi\right)=(\nabla-i \mathbf{A})^{2} \psi+\left(1-|\psi|^{2}\right) \psi, \\
\Delta \varphi=\operatorname{div}\left\{\operatorname{Im}\left[\psi^{*}(\nabla-\mathrm{iA}) \psi\right]\right\},
\end{gathered}
$$

In Eqs. (1a) and (1b) all the physical quantities (order parameter $\psi=|\psi| e^{i \phi}$, vector potential $\mathbf{A}$, and electrostatical poten-

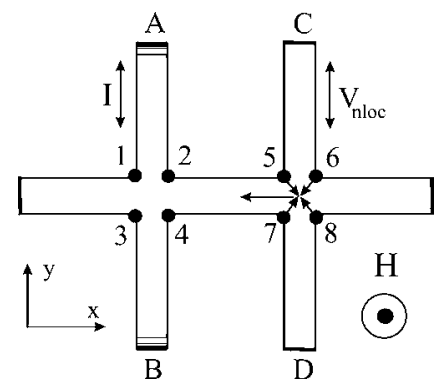

FIG. 1. Schematic diagram of the Hall-shaped superconductor. Arrows in the cross of lead $C D$ show the direction of motion of vortices penetrating the sample through points 5-8 (see text below). 
tial $\varphi$ ) are measured in dimensionless units: The vector potential $\mathbf{A}$ and momentum of superconducting condensate $\mathbf{p}=\nabla \phi-\mathbf{A}$ is scaled in units of $\Phi_{0} /(2 \pi \xi)$ (where $\Phi_{0}$ is the quantum of magnetic flux), the order parameter is in units of $\Delta_{0}$, and the coordinates are in units of the coherence length $\xi(T)$. In these units the magnetic field is scaled by $H_{c 2}$ and the current density by $j_{0}=c \Phi_{0} / 8 \pi^{2} \lambda^{2} \xi$. Time is scaled in units of the Ginzburg-Landau relaxation time $\tau_{\mathrm{GL}}=\pi \hbar / 8 k_{B}\left|T_{c}-T\right| u$, the electrostatic potential $(\varphi)$ is in units of $\varphi_{0}=\hbar / 2 e \tau_{\mathrm{GL}}$.

We neglected screening effects that allow us to neglect the equation for the vector potential $\mathbf{A}=(0,-H x, 0)$. This is allowed because in the experiment ${ }^{17}$ usually the size of the system was much smaller than $\lambda_{\text {eff }}=\lambda^{2} / d$. In our numerical calculations we took the width of all stripes equal to $10 \xi$ and the size of the structure in the $x$ direction be $140 \xi$ and $90 \xi$ in the $y$ direction (see Fig. 1). At the ends of the $A-B$ lead we used $N-S$ boundary conditions (in order to inject the current into the superconductor). At the other boundaries we used superconductor-vacuum boundary conditions. In the experiment the ends of the structure are connected by wide superconductors [see Fig. 1(a) in Ref. 17]. As a consequence vortices have the possibility to come and leave our sample from the wide contacts. At high magnetic fields the vortex lattice is softer in the wide superconductors and the local disturbance will decay within several intervortex distances. ${ }^{17}$ In other words, if a vortex wants to leave the narrow sample and enter the wide one it should be prohibited because of the presence of plenty of vortices in the contacts which repel them. To model both situations we considered two cases: (i) "open ends" case and (ii) "close ends" case. In the "open ends" case we apply the variation of the critical temperature in the end of the leads of the Hall-shaped superconductor

$$
T_{c}(r)= \begin{cases}T_{c 0} & r>10, \\ T_{c 0} r / 10 & 0<r<10,\end{cases}
$$

where $r$ is the distance from the lead's ends. In the "close ends" case there is no variation of the critical temperature. It turned out that in contrast to the "close ends" case in the "open ends" model the vortices may freely leave or enter the sample if the number of vortices is far from the equilibrium value for the given value of the magnetic field. It drastically changes the response of the system on the applied current. In Fig. 2 we presented local and nonlocal voltage response for these two cases. For the "open ends" model the nonlocal voltage response is almost symmetrical with respect to the sign of the applied current (at high magnetic fields) while for "close ends" there is a pronounced rectify effect. We explain that difference by the influence of the surface barrier. Indeed, the magnetization curve for the "open ends" model exhibits hysteresis only at low magnetic fields while for the "close ends" model, the hysteresis exist in a much larger field region (see Fig. 3). Vortex distribution for sweeping up and sweeping down the magnetic field illustrates the effect of the surface barrier (Figs. 4 and 5). For the "open ends" model (Fig. 4) the number of the vortices is almost the same for increasing and decreasing the magnetic field while for the "close ends" case (Fig. 5) their number differs considerably.

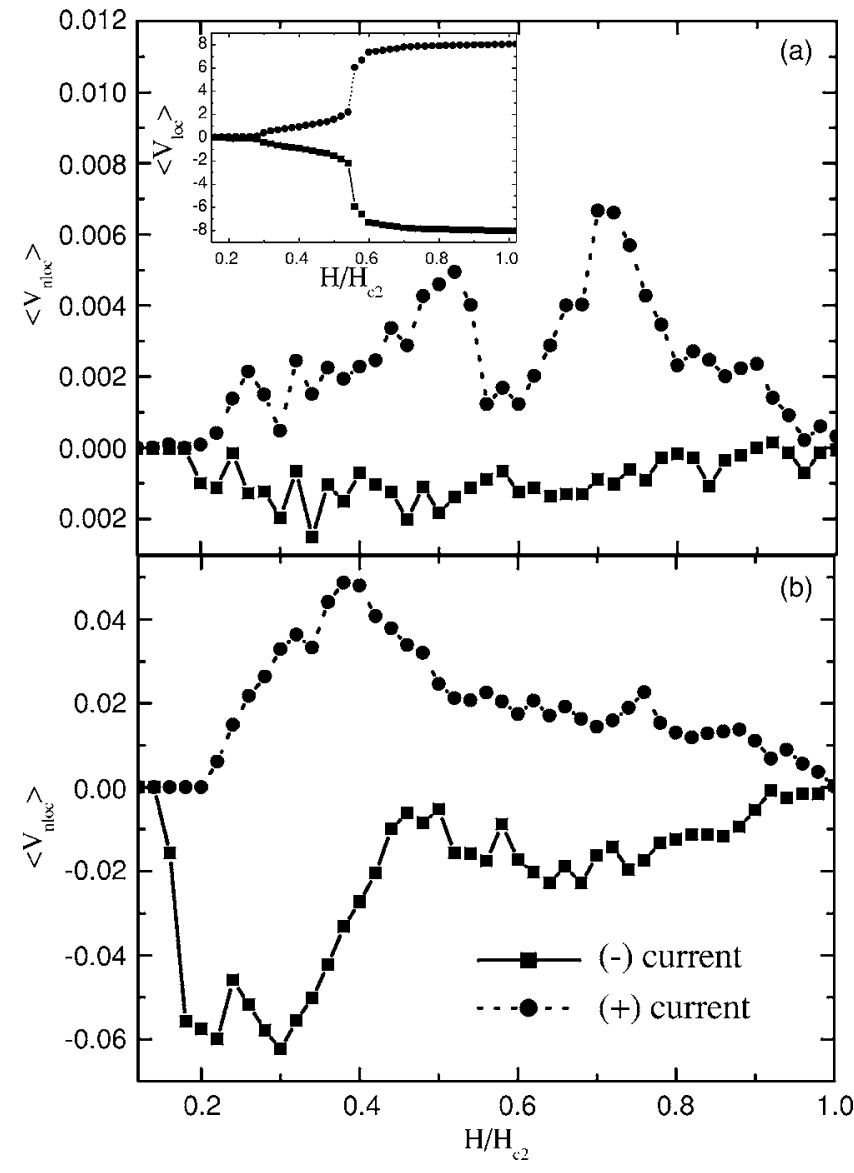

FIG. 2. Magnetic field dependence of the nonlocal voltage $(C D)$ for different directions of the injected current $\left(j=0.1 j_{0}\right)$ in the $A-B$ lead for cases of the "close ends" (a) and "open ends" (b) models. Notice that there are not only qualitative differences (no rectify effect for "open ends" model at large magnetic fields) but also quantitative one-weaker nonlocal voltage for "close ends" model. In the inset the dependence of the local voltage $(A B)$ on $H$ is shown (for "open ends" model local voltage response is the same). $V_{\text {loc }}$ appears first at $H \simeq 0.16$ because of vortex flow through the corners 1-4 (see Fig. 1). Sharp increase in $V_{\text {loc }}$ at $H=0.3$ is connected with vortex flow along the whole $A-B$ lead and at $H=0.56$ with the transition of the $A-B$ lead to the normal state.

Below we show in detail how the influence of the surface barrier leads to the rectify effect. From now we restrict our discussion to the Hall-shaped superconductor with "close ends."

When we apply the current along the $A B$ lead it acts on the vortices through the Lorentz force $\Phi_{0}[\mathbf{n} \times \mathbf{j}] / c$ which leads to a force directing the vortices to leave the lead. But the vortices in the neighborhood will prevent that motion. The vortices are prevented to leave the sample through the edges because of the presence of a surface barrier (see Refs. 20-23 for a discussion on the effect of the surface barrier on the critical current). As a result, for currents less than the critical one (which actually depends on the number of vortices and hence on the value of the magnetic field) the local and nonlocal voltage drop is equal to zero. In Fig. 6 we present the vortex distribution at $H=0.2$ and for $j=I / w d=0.08 j_{0}$ ( $w$ is the width and $d$ is the thickness of the 


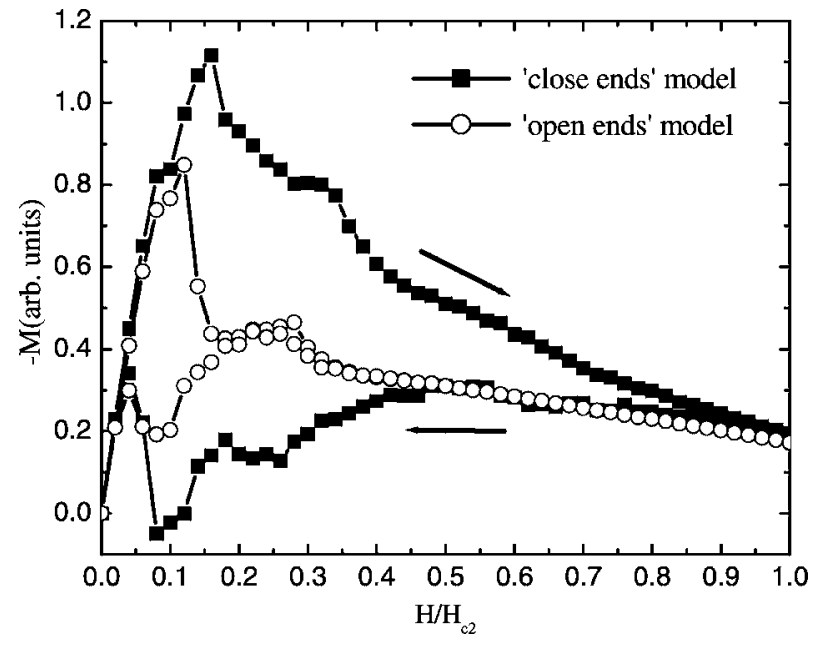

FIG. 3. Magnetization curves of the Hall-shaped superconductor with "close" and "open" ends are obtained by sweeping the magnetic field up and down from $H=0$ to $H=H_{c 2}$. At $H \geqslant 0.2 H_{c 2}$ the $M(H)$ is almost reversible for "open ends" model.

superconducting leads) just below the critical value. Throughout the paper we call $I_{-}$the current which pushes the vortices to the direction of the $C D$ contact and $I_{+}$which pulls the vortices in the opposite direction (at the given direction of the external magnetic field).

Comparison with Fig. 5 clearly shows that the current in the $A B$ lead indeed drives the vortex system in the $C D$ lead to the critical state. The larger the value of the injected current the further the vortex configuration in the $C D$ lead from equilibrium.

When the current in the $A B$ lead exceeds some critical value $I_{c}$ (which depends on the applied magnetic field) the vortex/flux flow regime starts. Because of the presence of
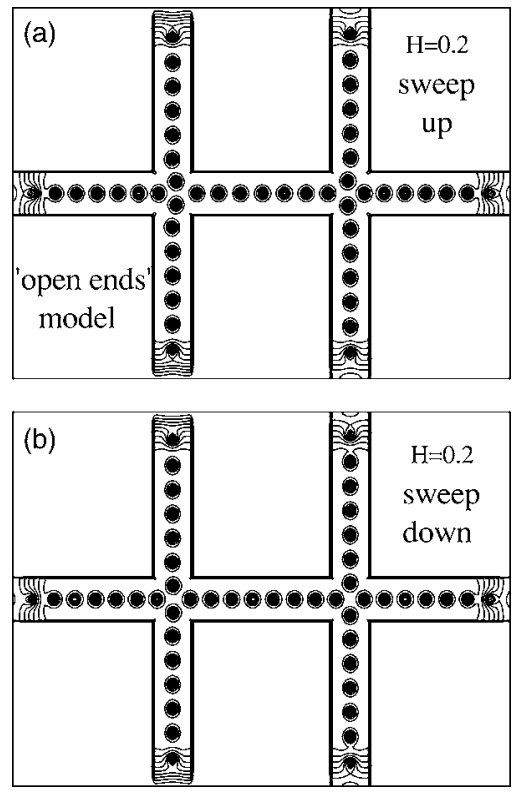

FIG. 4. Vortex configurations for sweeping up (a), sweeping down (b) magnetic field for the case of "open ends" model. There is no injected current in the system.
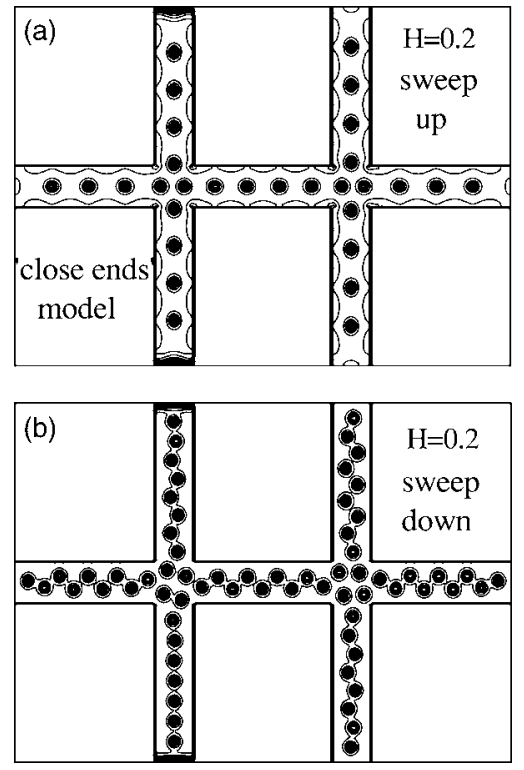

FIG. 5. Vortex configurations for sweeping up (a), sweeping down (b) magnetic field for the case of "close ends" model. There is no injected current in the system.

corners in our geometry (points 1-4 in Fig. 1) vortex flow first occurs through such sharp corners where the surface barrier is first suppressed (the same is found when the corners are sharp on a length scale determined by $\xi$ ). With increasing applied current, vortices start to penetrate/leave the sample in other points (e.g., the sides of the sample) which leads to a sharp increase in the local voltage. A similar behavior is found if we keep the value of the injected current constant and change the magnetic field [see the inset of Fig. 2(a)].

A nonlocal voltage $(C D)$ appears at currents larger than the critical one. In Fig. 2(a) we show the dependence of the nonlocal voltage on the applied magnetic field at a fixed value of the injected current $j=I / w d=0.1 j_{0}$. At currents just above $I_{c}(H)$ the nonlocal voltage $V_{\text {nloc }}(t) \neq 0$ with a timeaveraged $\left\langle V_{\text {nloc }}\right\rangle=0$. The vortices only tremble in the $C D$ lead as a consequence of the changing number of vortices in the $A B$ lead due to the entrance/exit of vortices in this lead. It is necessary to increase further the injected current in order to observe a nonzero $\left\langle V_{\text {nloc }}\right\rangle$. Our calculation shows that the nonlocal voltage drop is always connected with the entrance/ exit of vortices through the corners of lead $C D$ (points 5-8 in Fig. 1).

We should note that $\left\langle V_{\text {nloc }}\right\rangle$ depends on the direction of the injected current. In Fig. 7 we present the time dependence of $\mathrm{V}_{\text {nloc }}(t)$ at $H=0.5$. Every peak in Fig. 7 corresponds to the entrance or exit of vortices in the corners of the $C$ - $D$ stripe. For (+) direction of the current, a positive peak corresponds to the entrance of vortices through points 6 and 8 , and a negative peak to the entrance of vortices through points 5 and 7. The latter process does not contribute to the timeaveraged $\left\langle V_{\text {nloc }}\right\rangle$ because it is compensated by the subsequent motion of the vortices in the direction of the $A-B$ lead. For the $(-)$ direction of the current, positive peaks correspond to the exit of vortices through points 5 and 7 and negative peaks 


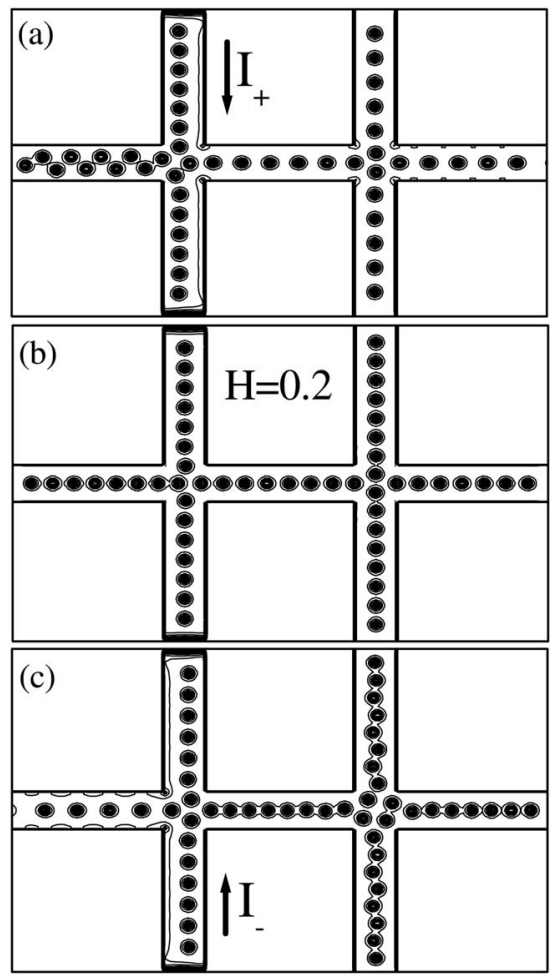

FIG. 6. Vortex distribution in the Hall-shaped superconductor with nonzero (a),(c), and zero (b) injected current. The value of the current $I_{ \pm}$was taken just below the critical one. Vortex distribution in (b) case was found by starting from the initial condition $\psi=0$ everywhere which models the field cooled condition.

to the exit of vortices through points 6 and 8 . In the former case, this process also does not give a contribution to $\left\langle V_{\text {nloc }}\right\rangle$ because the vortices were coming from the $A-B$ lead. So only the passage of the vortices through the cross in the $C-D$ stripe (they should enter or exit via points 6 and 8 - see Fig. 1) lead to a nonzero contribution to $\left\langle V_{\text {nloc }}\right\rangle$. This is connected with the fact that the time-averaged voltage in the $y$ direction is proportional to the integrated displacement of the vortex in the $x$ direction. For vortices penetrating or leaving through points 5 and 7 this displacement is equal to zero (see Fig. 1). From Fig. 7 it is clearly seen that vortices more easily enter the $C$ - $D$ lead [Fig. 7(a)] than leave it [number of peaks is large for $(+)$ current than for $(-)$ one]. This seems to be a general property of the investigated system when one injects current in the $A-B$ lead and we connect this with the presence of the surface barrier.

The nonlocal voltage $\left\langle V_{\text {nloc }}\right\rangle$ is also a quite nonmonotonous function with the applied magnetic field for a fixed value of the applied current. As was discussed in Ref. 17 this is connected with the small size and geometry of our system. Changing the magnetic field crucially changes the number of vortices and the vortex structure in our sample. For a large system we can expect that the response of the vortex lattice should gradually increase with growing $H$ at low magnetic fields and at $H \sim H_{c 2}$ it approaches zero, as shown in Fig. 3(a) of Ref. 17. The reason is that when the vortex lattice is close to a triangle one, with changing $H$ only the lattice parameter varies. But, in our system a change of $H$ also leads

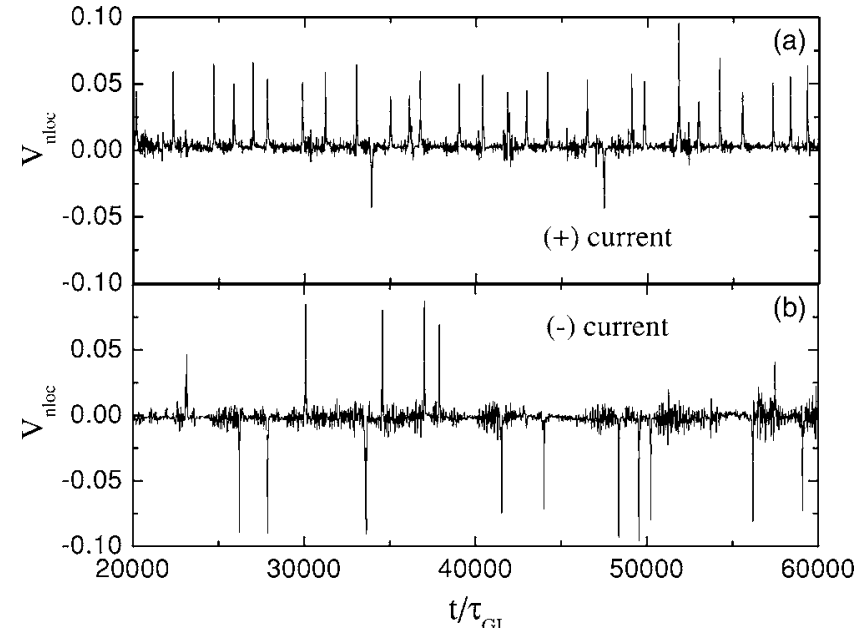

FIG. 7. Time dependence of the nonlocal voltage for (+) (a) and (-) (b) directions of the injected current at $H=0.5$.

to a change of the arrangement of vortices. This influences the vortex entry/exit process in the cross area. At different $H$ it becomes easier or harder to push(pull) vortices in(out) of the $C-D$ stripe and it also changes the "channels" via which vortices enter/leave the sample. In Fig. 8 we show the vortex structure and in Fig. 9 the nonlocal voltage response for two values of the magnetic fields. For $H=0.52$ vortices mainly enter through the corners 6 and 8 and $\left\langle V_{\text {nloc }}\right\rangle$ is relatively large. But at $H=0.56$ the majority of the vortices enter through the corners 5 and 6 and $\left\langle V_{\text {nloc }}\right\rangle$ is small because these vortices do not contribute to the time-averaged nonlocal voltage.

This led us to believe that the surface barrier is responsible for the dependence of the nonlocal voltage on the di-
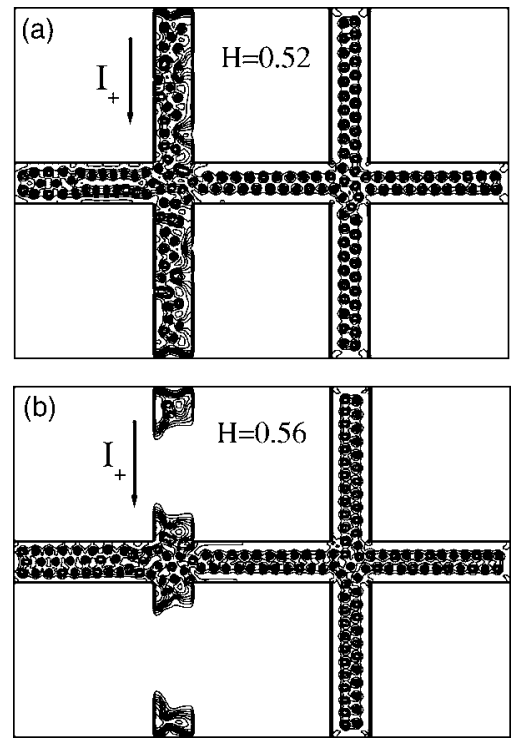

FIG. 8. Snapshot of the vortex distribution in a Hall-shaped superconductor at $H=0.52$ (a) and $H=0.56$ (b). Applied current $(j=0.1)$ with $(+)$ direction in $A-B$ lead exceeds the critical value and it leads to flux flow. At $H=0.56$ part of the $A-B$ lead is in the normal state. 


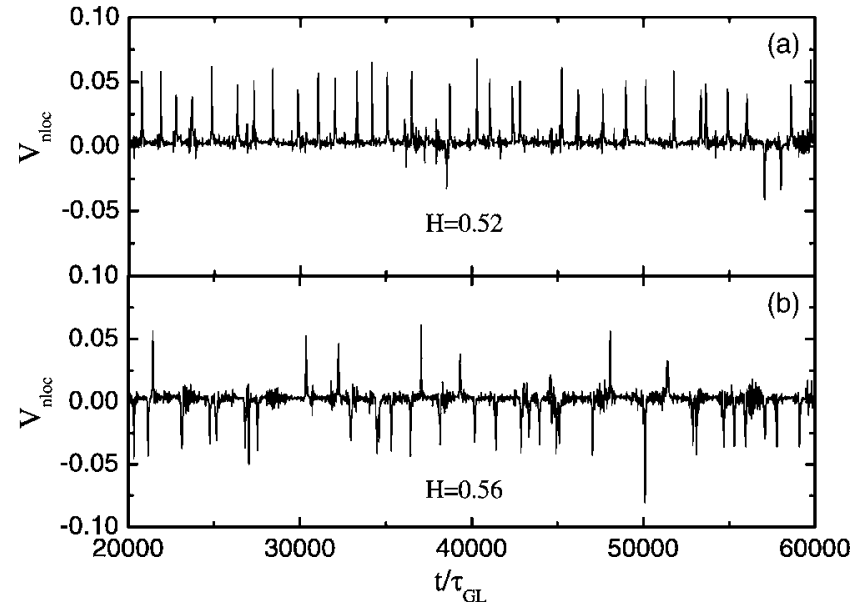

FIG. 9. Time dependence of the nonlocal voltage for the vortex structures of Fig. 8.

rection of the current. It is interesting to note that the local voltage is direction independent (see insert in Fig. 5). This originates from the fact that the number of vortices in the $A-B$ stripe does not depend on the direction of the applied current (see Fig. 6).

In conclusion, the physical reason for the occurrence of nonlocal effects in Hall-shaped mesoscopic superconductors is the intervortex interaction. Besides due to the specific geometry of the system and the presence of the surface barrier the nonlocal voltage response is nonmonotonous with a changing magnetic field and it depends on the direction of the applied current. This is mainly connected with the com- plexity and the small (compared to the coherence length) width of the leads of our structure. As a result the property of the quasi-one-dimensional vortex lattice may change drastically with a small change of the number of vortices. We showed that the surface barrier plays a crucial role in the above phenomena. This is connected with the asymmetry in the vortex entry and exit in superconductors where the effect of the surface barrier is important. ${ }^{24-26}$ In our geometry this asymmetry is enhanced by the presence of corners (points 1-8 in Fig. 1). We did not study dependence of the nonlocal voltage on the distance $L$ between current and voltage leads or on the width $w$ of the connected lead. But it is obvious that in the absence of bulk pinning the nonlocal voltage response should exist at any $L$ and $w$ because in such a system a local perturbation will spread over an infinitive distance in case of "open ends" model.

In our previous study ${ }^{17}$ of superconducting Hall bar structures nonlocal response was found experimentally and explained theoretically. No rectifying effect was observed. Therefore we may conclude that in this case the "open ends" model is more appropriate. Probably, in the experiment the surface barrier is almost suppressed in the cross regions (due to surface defects, fluctuations of temperature, or other reasons) and vortices may almost freely enter/leave the sample if their number is far from the equilibrium value.

This work was supported by the Belgian Science Policy, GOA (University of Antwerp), the ESF-network on "Vortex matter," and the Flemish Science Foundation (FWO-Vl) and EPSRC (UK). One of us (D.Y.V.) is supported by the INTAS Young Scientist Fellowship 04-83-3139.
*Electronic address: francois.peeters@ua.ac.be

${ }^{1}$ J. E. Villegas, S. Savel'ev, F. Nori, E. M. Gonzalez, J. V. Anguita,

R. Garcia, and J. L. Vicent, Science 302, 1188 (2003).

${ }^{2}$ G. Carapella and G. Costabile, Phys. Rev. Lett. 87, 077002 (2001).

${ }^{3}$ R. Wordenweber, P. Dymashevski, and V. R. Misko, Phys. Rev. B 69184504 (2004).

${ }^{4}$ J. Van de Vondel, C. C. de Souza Silva, B. Y. Zhu, M. Morelle, and V. V. Moshchalkov, Phys. Rev. Lett. 94, 057003 (2005).

${ }^{5}$ D. Lopez, W. K. Kwok, H. Safar, R. J. Olsson, A. M. Petrean, L. Paulius, and G. W. Crabtree, Phys. Rev. Lett. 821277 (1999).

${ }^{6}$ Yu. Eltsev, K. Nakao, S. Shibata, and N. Koshizuka, Physica C 341-348, 1107 (2000).

${ }^{7}$ J. H. S. Torres, R. Ricardo da Silva, S. Moehlecke, and Y. Kopelevich, Solid State Commun. 125, 11 (2003).

${ }^{8}$ C. S. Lee, B. Janko, I. Derenyi, and A. L. Barabasi, Nature (London) 400, 337 (1999).

${ }^{9}$ J. F. Wambaugh, C. Reichhardt, C. J. Olson, F. Marchesoni, and F. Nori, Phys. Rev. Lett. 83, 5106 (1999).

${ }^{10}$ C. J. Olson, C. Reichhardt, B. Janko, and F. Nori, Phys. Rev. Lett. 87, 177002 (2001).

${ }^{11}$ B. Y. Zhu, F. Marchesoni, and F. Nori, Phys. Rev. Lett. 92, 180602 (2004).

${ }^{12}$ B. Y. Zhu, F. Marchesoni, V. V. Moshchalkov, and F. Nori, Phys.
Rev. B 68, 014514 (2003)

${ }^{13}$ S. Savel'ev and F. Nori, Nat. Mater. 1, 179 (2002).

${ }^{14}$ S. Savel'ev, F. Marchesoni, and F. Nori, Phys. Rev. Lett. 91, 010601 (2003).

${ }^{15}$ S. Savel'ev, F. Marchesoni, and F. Nori, Phys. Rev. Lett. 92, 160602 (2004)

${ }^{16}$ A. V. Ustinov, C. Coqui, A. Kemp, Y. Zolotaryuk, and M. Salerno, Phys. Rev. Lett. 93, 087001 (2004).

${ }^{17}$ I. V. Grigorieva, A. K. Geim, S. V. Dubonos, K. S. Novoselov, D. Y. Vodolazov, F. M. Peeters, P. H. Kes, M. Hesselberth, and M. G. Blamire, Phys. Rev. Lett. 92, 237001 (2004).

${ }^{18}$ P. L. McEuen, A. Szafer, C. A. Richter, B. W. Alphenaar, J. K. Jain, A. D. Stone, R. G. Wheeler, and R. N. Sacks, Phys. Rev. Lett. 64, 2062 (1990).

${ }^{19}$ A. K. Geim, P. C. Main, P. H. Beton, P. Streda, L. Eaves, C. D. W. Wilkinson, and S. P. Beaumont, Phys. Rev. Lett. 67, 3014 (1991).

${ }^{20}$ M. Yu. Kupriyanov and K. K. Likharev, Fiz. Tverd. Tela (Leningrad) 16, 2829 (1974) [Sov. Phys. Solid State 16, 1835 (1975)].

${ }^{21}$ L. G. Aslamazov and S. V. Lempickii, Zh. Eksp. Teor. Fiz. 84, 2216 (1983) [Sov. Phys. JETP 57, 1291 (1983)].

${ }^{22}$ M. Benkraouda and J. R. Clem, Phys. Rev. B 58, 15103 (1998).

${ }^{23}$ B. L. T. Plourde, D. J. Van Harlingen, D. Yu. Vodolazov, R. Besseling, M. B. S. Hesselberth, and P. H. Kes, Phys. Rev. B 64, 
014503 (2001).

${ }^{24}$ J. R. Clem, in Proceedings of 13th Conference on Low Temperature Physics (LT 13), edited by K. D. Timmerhaus, W. J. O’Sullivan, and E. F. Hammel (Plenum, New York, 1974), Vol.
3, p. 102.

${ }^{25}$ L. Burlachkov, Phys. Rev. B 47, 8056 (1993).

${ }^{26}$ D. Y. Vodolazov, I. L. Maksimov, and E. H. Brandt, Physica C 384, 211 (2003). 